\title{
Chronic kidney disease in Taiwan
}

\author{
Pierre Delanaye $^{\mathrm{a}}$, Etienne Cavalier ${ }^{\mathrm{b}}$, Jean-Marie Krzesinski ${ }^{\mathrm{a}}$ \\ ${ }^{a}$ Department of Dialysis-Nephrology ; ${ }^{b}$ Department of Clinical Chemistry, University of Liège, CHU Sart Tilman, 4000 Liège, Belgium
}

In Chi Peng Wen and colleagues' paper on the mortality attributable to chronic kidney disease (CKD) in Taiwan, ${ }^{1}$ the prevalence of CKD must be interpreted with caution.

Wen and colleagues use a non-calibrated creatinine concentration to estimate glomerular filtration rate (GFR). Even if such non-calibration has little importance for mortality linked to CKD, it could have serious consequences on prevalence data. ${ }^{2}$ These prevalence data are thus not easy to compare with those of the US population, for which the newly expressed Modification of Diet in Renal Disease (MDRD) study equation ${ }^{3}$ was used. This equation is applied to standardised creatinine with a factor of 175; Wen and colleagues used the version of the equation with a factor of 186.

Moreover, the relation between creatinine and GFR varies with ethnic origin. For African-American ${ }^{3}$ and Japanese $^{4}$ populations, correction factors of 1.21 and 0.763 , respectively, must be applied. Such a correction was not done by Wen and colleagues and should be discussed.

Lastly, Wen and colleagues showthat nearly one in three patients older than 65 years presented with stage 3 CKD. The term "disease" in this age group is debatable because the normal GFR in older populations is not well defined; a GFR of less than $60 \mathrm{~mL} / \mathrm{min} / 1.73 \mathrm{~m}^{2}$ could be regarded as physiological in a healthy older person. ${ }^{5}$

\section{References}

1 Wen CP, Cheng TYD, Tsai MK, et al. All-cause mortality attributable to chronic kidney disease: a prospective cohort study based on 462293 adults in Taiwan. Lancet 2008; 371: 2173-82.

2 Coresh J, Eknoyan G, Levey AS. Estimating the prevalence of low glomerular filtration rate requires attention to the creatinine assay calibration. J Am Soc Nephrol 2002; 13: 2811-12.

3 Levey AS, Coresh J, Greene T, et al. Using standardized serum creatinine values in the Modification of Diet in Renal Disease study equation for estimating glomerular filtration rate. Ann Intern Med 2006; 145: 24754.

4 Imai E, Horio M, Nitta K, et al. Modification of the Modification of Diet in Renal Disease (MDRD) Study equation for Japan. Am J Kidney Dis 2007; 50: 927-37.

5 Glassock RJ, Winearls C. An epidemic of chronic kidney disease: fact or fiction? Nephrol Dial Transplant 2008; 23:1117-21. 